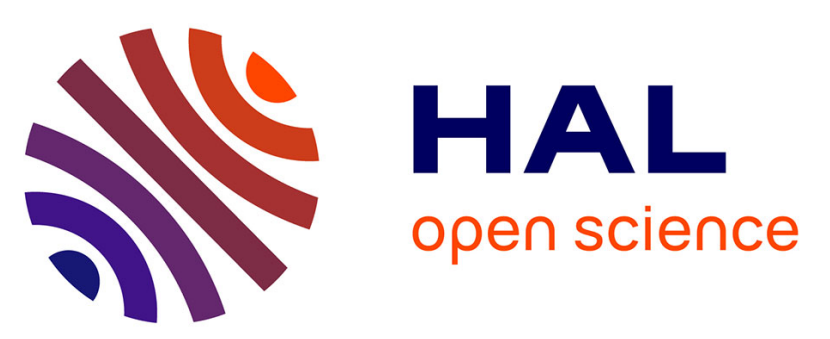

\title{
Conditional extremes from heavy-tailed distributions: An application to the estimation of extreme rainfall return levels
}

Laurent Gardes, Stéphane Girard

\section{To cite this version:}

Laurent Gardes, Stéphane Girard. Conditional extremes from heavy-tailed distributions: An application to the estimation of extreme rainfall return levels. 2009. hal-00371757v1

HAL Id: hal-00371757

https://hal.science/hal-00371757v1

Preprint submitted on 30 Mar 2009 (v1), last revised 23 Apr 2013 (v4)

HAL is a multi-disciplinary open access archive for the deposit and dissemination of scientific research documents, whether they are published or not. The documents may come from teaching and research institutions in France or abroad, or from public or private research centers.
L'archive ouverte pluridisciplinaire HAL, est destinée au dépôt et à la diffusion de documents scientifiques de niveau recherche, publiés ou non, émanant des établissements d'enseignement et de recherche français ou étrangers, des laboratoires publics ou privés. 


\title{
CONDITIONAL EXTREMES FROM HEAVY-TAILED DISTRIBUTIONS: AN APPLICATION TO THE ESTIMATION OF EXTREME RAINFALL RETURN PERIODS
}

\author{
Laurent Gardes and Stéphane Girard* \\ Team Mistis, INRIA Rhône-Alpes \& Laboratoire Jean Kuntzmann \\ 655, avenue de l'Europe, Montbonnot, 38334 Saint-Ismier Cedex, France. \\ * Corresponding author, Stephane.Girard@inrialpes.fr
}

\begin{abstract}
This paper is dedicated to the estimation of extreme quantiles and tail index from heavy-tailed distributions when a covariate is recorded simultaneously with the quantity of interest. A nearest neighbor approach is used to construct our estimators. Their asymptotic normality is established under mild regularity conditions. An application to the estimation of return periods of extreme rainfalls in the Cévennes-Vivarais region is provided.
\end{abstract}

Keywords - Conditional extreme quantiles, heavy-tailed distribution, nearest neighbor estimator, extreme rainfalls.

AMS Subject classifications - 62G32, 62G05, 62E20.

\section{Introduction}

An important literature is dedicated to the estimation of extreme quantiles, i.e. quantiles of order $1-\alpha$ with $\alpha$ tending to zero as the sample size increases. The most popular estimator was proposed by Weissman [31], in the context of heavy-tailed distributions, and adapted to Weibull-tail distributions in [14, 17]. We also refer to [13] for the general case.

When some covariate $x$ is recorded simultaneously with the quantity of interest $Y$, the extreme quantile thus depends on the covariate and is referred in the sequel to as the conditional extreme quantile. In our real data study, we are interested in the estimation of return periods associated to extreme rainfalls as a function of the geographical location. In this case, $x$ is a three-dimensional covariate involving the longitude, latitude and altitude.

Parametric models for conditional extremes are proposed in $[12,29]$ whereas semi-parametric methods are considered in [2, 22]. Fully non-parametric estimators have been first introduced in [11], where a local polynomial modelling of the extreme observations is used. Similarly, spline estimators are fitted in [6] through a penalized maximum likelihood method. In both cases, the authors focus on univariate covariates and on the finite sample properties of the estimators. These results are extended in [3] where local polynomials estimators are 
proposed for multidimensional covariates and where their asymptotic properties are established.

We propose here to estimate the conditional extreme quantile by a nearest neighbor approach. We refer to [25] for the first asymptotic properties of the nearest neighbor density estimator and to [30] for the regression case. As an illustration, in the above mentioned climatology study, the estimation of the return period at a given geographical point is based on rainfalls measured at the nearest raingauges. Once the selection of the nearest observations is achieved, extreme-value methods are used to estimate the conditional quantile. Whereas no parametric assumption is made on the covariate $x$, we assume that the conditional distribution of $Y$ given $x$ is heavy-tailed. This semi-parametric assumption amounts to supposing that the conditional survival function decreases at a polynomial rate. The conditional tail index $\gamma(x)$ drives this rate of convergence and has to be estimated before conditional extreme quantiles. In our real data study, the estimation of $\gamma(x)$ permits to assess the tail-heaviness of the rainfall distribution at each geographical point $x$, indicating which areas are more likely to suffer from extreme climate events.

Nearest neighbor estimators of the conditional tail-index and conditional extreme quantiles are defined in Section 2. Their asymptotic distributions are derived in Section 3. Some examples are provided in Section 4 and the application to the extreme rainfall study is presented in Section 5. Proofs are postponed to Section 6 .

\section{Nearest neighbor estimators}

Let $E$ be a metric space associated to a metric $d$. For $y>0$ and $x \in E$, denote by $F(y, x)$ the conditional distribution function of $Y$ given $x$. For instance, in the case where $E$ is finite dimensional, each coordinate of $x$ may represent a geographical coordinate. At the opposite, when $x$ is a time series or a curve, $E$ is infinite dimensional. We assume that for all $x \in E$, the conditional distribution function of $Y$ is heavy-tailed. More specifically, we have for all $y>0$,

$$
1-F(y, x)=y^{-1 / \gamma(x)} L(y, x),
$$

or equivalently, for all $\alpha \in(0,1]$,

$$
q(\alpha, x)=F^{\leftarrow}(1-\alpha, x)=\alpha^{-\gamma(x)} \ell\left(\alpha^{-1}, x\right),
$$

where $F^{\leftarrow}(1-\alpha, x)=\sup \{y>0, F(y, x) \leq 1-\alpha\}$ denotes the generalized inverse of $F(., x)$. Here, $\gamma($.$) is an unknown positive function of the covariate x$ referred to as the conditional tail index. The larger $\gamma(x)$ is, the heavier is the tail at point $x$. Besides, for all $x \in E$ fixed, $L(., x)$ and $\ell(., x)$ are slowly varying functions, i.e. for all $v>0$,

$$
\lim _{y \rightarrow \infty} \frac{L(v y, x)}{L(y, x)}=\lim _{y \rightarrow \infty} \frac{\ell(v y, x)}{\ell(y, x)}=1 .
$$

Let $\left(Y_{1}, x_{1}\right), \ldots,\left(Y_{n}, x_{n}\right)$ be a sample of independent observations from (1). For a given $t \in E$, our aim is to build an estimator of $\gamma(t)$ and, for a sequence $\alpha_{n, t}$ tending to 0 as $n$ goes to infinity, an estimator of $q\left(\alpha_{n, t}, t\right)$. In the sequel, $q\left(\alpha_{n, t},.\right)$ is referred to as a conditional extreme quantile. As an example, if 
$Y_{1}, \ldots, Y_{n}$ are independent rainfalls collected during a period of $T$ years in $n$ sites characterized by their geographical positions $x_{1}, \ldots, x_{n}$, then, $q\left(T /\left(n T^{\prime}\right), t\right)$ is the $T^{\prime}$ years return period at position $t$. In other words, $q\left(T /\left(n T^{\prime}\right), t\right)$ represents the rainfall observed approximatively every $T^{\prime}$ years. Here, we focus on the case where the design points $x_{1}, \ldots, x_{n}$ are non random. Let $m_{n, t}$ be a sequence of integers such that $1<m_{n, t}<n$ and let $\left\{x_{1}^{*}, \ldots, x_{m_{n, t}}^{*}\right\}$ be the $m_{n, t}$ nearest covariates of $t$ (with respect to the distance $d$ ). The associated observations taken from $\left\{Y_{1}, \ldots, Y_{n}\right\}$ are denoted by $\left\{Z_{1}, \ldots, Z_{m_{n, t}}\right\}$. The corresponding order statistics are denoted $Z_{1, m_{n, t}} \leq \ldots \leq Z_{m_{n, t}, m_{n, t}}$ and the rescaled logspacings are defined as:

$$
C_{i, n, t} \stackrel{\text { def }}{=} i \log \left(\frac{Z_{m_{n, t}-i+1, m_{n, t}}}{Z_{m_{n, t}-i, m_{n, t}}}\right) .
$$

Our estimators of $\gamma(t)$ are linear combinations of these rescaled log-spacings:

$$
\hat{\gamma}_{n}(t, a, \lambda)=\sum_{i=1}^{k_{n, t}} p\left(\frac{i}{k_{n, t}}, a, \lambda\right) C_{i, n, t} / \sum_{i=1}^{k_{n, t}} p\left(\frac{i}{k_{n, t}}, a, \lambda\right),
$$

where $k_{n, t}$ is a sequence of integers such that $1<k_{n, t}<m_{n, t}$ and the weights are defined for all $s \in(0,1), a \geq 1,0<\lambda \leq 1$ by

$$
p(s, a, \lambda)=\frac{\lambda^{-a}}{\Gamma(a)} s^{1 / \lambda-1}(-\log (s))^{a-1} .
$$

Note that $p(., a, \lambda)$ is the density function on $(0,1)$ introduced as the log-gamma distribution by [9]. Examples of such densities are provided in Section 4 and illustrated on Figure 1. Four main behaviors can be exhibited: (i) $p(., 1,1)$ is constant, (ii) $p(., 1, \lambda)$ is increasing for all $0<\lambda<1$, (iii) $p(., a, 1)$ is decreasing for all $a>1$ and (iv) $p(s, a, \lambda)$ has an unique mode at $s=\exp \{\lambda(1-a) /(1-\lambda)\}$ for $a>1$ and $0<\lambda<1$.

In the same spirit as the quantile estimator proposed by Weissman [31], the following estimator of $q\left(\alpha_{n, t}, t\right)$ can be derived from (4):

$$
\hat{q}\left(\alpha_{n, t}, t\right)=Z_{m_{n, t}-k_{n, t}+1, m_{n, t}}\left(\frac{k_{n, t}}{m_{n, t} \alpha_{n, t}}\right)^{\hat{\gamma}_{n}(t, a, \lambda)},
$$

where $\alpha_{n, t}$ is a sequence in $(0,1)$. The limiting distributions of these estimators are established in the next section.

\section{$3 \quad$ Asymptotic results}

We first give all the conditions and notations required to obtain the asymptotic normality of our estimators. In the sequel, we fix $t \in E$ such that $\gamma(t)>0$.

(A.1) The slowly varying function $\ell(., t)$ is normalized.

Assumption (A.1) is equivalent to supposing that, for $\alpha \in(0,1)$, the Karamata representation of $q(\alpha, t)$ can be simplified as:

$$
q(\alpha, t)=c(t) \exp \left\{\int_{1}^{\alpha^{-1}} \frac{\gamma(t)+\Delta(v, t)}{v} d v\right\},
$$


with $c(t)>0$ and where $\Delta(v, t)$ converges to 0 as $v$ goes to infinity. Note also that condition (A.1) implies (1), see for instance [4] or [18]. The next two assumptions control the rate of convergence of the function $\Delta(., t)$ to zero.

(A.2) The function $\Delta(., t)$ is regularly varying with index $\rho(t)<0$, i.e. for all $v>0$

$$
\lim _{y \rightarrow \infty} \frac{\Delta(v y, t)}{\Delta(y, t)}=v^{\rho(t)} .
$$

Conditions (A.1) and (A.2) imply that for all $v>0$,

$$
\log \left(\frac{\ell(v y, t)}{\ell(y, t)}\right)=\Delta(y, t) \frac{1}{\rho(t)}\left(v^{\rho(t)}-1\right)(1+o(1)),
$$

which is the so-called second-order condition classically used to establish the asymptotic normality of tail-index estimators. The second-order parameter $\rho(t)$ controls the rate of convergence of $\Delta(v, t)$ to 0 i.e. the rate of convergence of $\ell(v y, t) / \ell(y, t)$ to 1 in equation (3). If $\rho(t)$ is close to 0 , this convergence is slow and thus the estimation of the conditional tail index and of the conditional extreme quantile are difficult.

(A.3) The function $|\Delta(., t)|$ is ultimately decreasing.

In the following, we denote by $\mathcal{V}_{n, t}$ the set $\left\{t, x_{1}^{*}, \ldots, x_{m_{n, t}}^{*}\right\} \subset E$. The largest oscillation of the log-quantile function with respect to its second variable is defined for all $\beta \in(0,1 / 2)$ as

$$
\omega_{n}(\beta)=\sup \left\{\left|\log \left(\frac{q(\alpha, x)}{q\left(\alpha, x^{\prime}\right)}\right)\right|, \alpha \in(\beta, 1-\beta),\left(x, x^{\prime}\right) \in \mathcal{V}_{n, t}^{2}\right\} .
$$

We also assume that $k_{n, t}$ is an intermediate sequence which is a classical assumption in extreme value theory.

(B) $m_{n, t} / k_{n, t} \rightarrow \infty$ and $k_{n, t} \rightarrow \infty$ as $n \rightarrow \infty$.

We are now in position to state our asymptotic normality result for $\hat{\gamma}_{n}(t, a, \lambda)$.

Theorem 1. Suppose (A.1), (A.2), (A.3) and (B) hold. If, moreover, for some $\delta>0$,

$$
k_{n, t}^{1 / 2} \Delta\left(m_{n, t} / k_{n, t}, t\right) \rightarrow \xi(t) \in \mathbb{R} \text { and } k_{n, t}^{2} \omega_{n}\left(m_{n, t}^{-(1+\delta)}\right) \rightarrow 0
$$

then

$$
k_{n, t}^{1 / 2}\left(\hat{\gamma}_{n}(t, a, \lambda)-\gamma(t)-\Delta\left(\frac{m_{n, t}}{k_{n, t}}, t\right) \mathcal{A B}(a, \lambda, \rho(t))\right)
$$

converges in distribution to a $\mathcal{N}\left(0, \gamma^{2}(t) \mathcal{A V}(a, \lambda)\right)$ random variable where

$$
\mathcal{A B}(a, \lambda, \rho(t))=(1-\lambda \rho(t))^{-a} \text { and } \mathcal{A} \mathcal{V}(a, \lambda)=\frac{\Gamma(2 a-1)}{\lambda \Gamma^{2}(a)}(2-\lambda)^{1-2 a} .
$$


The first part of condition (8) is standard in the extreme-value theory. It prevents the bias of the estimate from being too large compared to the standarddeviation. The second part of the condition is due to our conditional framework. It is dedicated to the control of the variations with respect to the covariate. For instance, if the slowly varying function $\ell$ does not depend on the covariate, the second part of condition (8) reduces to a regularity condition on the tail-index:

$$
k_{n, t}^{2} \log \left(m_{n, t}\right) \sup _{\left(x, x^{\prime}\right) \in \mathcal{V}_{n, t}^{2}}\left|\gamma(x)-\gamma\left(x^{\prime}\right)\right| \rightarrow 0 \text { as } n \rightarrow \infty .
$$

The following result establishes that $\hat{q}\left(\alpha_{n, t}, t\right)$ inherits its asymptotic distribution from $\hat{\gamma}_{n}(t, a, \lambda)$.

Theorem 2. Suppose (A.1), (A.2), (A.3), (B) and (8) hold. If, moreover, $\alpha_{n, t}<k_{n, t} / m_{n, t}$ then

$$
\frac{k_{n, t}^{1 / 2}}{\log \left(\frac{k_{n, t}}{m_{n, t} \alpha_{n, t}}\right)}\left(\log \left(\frac{\hat{q}\left(\alpha_{n, t}, t\right)}{q\left(\alpha_{n, t}, t\right)}\right)-\log \left(\frac{k_{n, t}}{m_{n, t} \alpha_{n, t}}\right) \Delta\left(\frac{m_{n, t}}{k_{n, t}}, t\right) \mathcal{A B}(a, \lambda, \rho(t))\right)
$$

converges in distribution to a $\mathcal{N}\left(0, \gamma^{2}(t) \mathcal{A} \mathcal{V}(a, \lambda)\right)$ random variable.

The asymptotic bias of estimators $\hat{\gamma}_{n}(., a, \lambda)$ and $\hat{q}\left(\alpha_{n, t},.\right)$ are both proportional to $\mathcal{A B}(a, \lambda, \rho(t))$ while their asymptotic variances are proportional to $\mathcal{A} \mathcal{V}(a, \lambda)$. These quantities can be controlled by an appropriate choice of $a$ and $\lambda$, see Section 4 for a discussion on this topic. Concerning the asymptotic variance, the proportionality factor is $\gamma^{2}(t)$. Hence, the heavier the tail is, the larger the asymptotic variance is. Moreover, the asymptotic variance can be lower bounded since

$$
\mathcal{A} \mathcal{V}(a, \lambda)-1=\int_{0}^{1}(p(s, a, \lambda)-1)^{2} d s \geq 0
$$

which entails that $\mathcal{A V}(a, \lambda) \geq 1$ for all $a \geq 1$ and $\lambda \in(0,1]$. It is thus clear that the minimum variance estimator is obtained with the uniform distribution $(a=\lambda=1)$. Let us also highlight that $\mathcal{A B}(a, \lambda, \rho(t))$ is an increasing function of $\rho(t)$. Thus, the closer $\rho(t)$ is to zero, the larger is the asymptotic bias. However, the second-order parameter $\rho(t)$ is unknown in practice making difficult the comparison of asymptotic bias associated to different log-gamma weights. We refer to $[20,21]$ for estimators of the second-order parameter in the unconditional case. To overcome this problem, one can define the mean-squared bias as:

$$
\mathcal{M S B}(a, \lambda)=\int_{-\infty}^{0} \mathcal{A B}^{2}(a, \lambda, \rho) d \rho=\frac{1}{\lambda(2 a-1)} .
$$

Note that the mean-squared bias converges to 0 as $a$ tends to infinity. It it thus not possible to define in our family a minimum mean-squared bias estimator. 


\section{Choice of log-gamma parameters}

\subsection{Nearest neighbor Hill estimator}

As remarked in the previous section, the minimum variance estimator is obtained by letting $a=\lambda=1$ in (5). This choice yields

$$
\hat{\gamma}_{n}^{\mathrm{H}}(t)=\hat{\gamma}_{n}(t, 1,1)=\frac{1}{k_{n, t}} \sum_{i=1}^{k_{n, t}} C_{i, n, t} .
$$

which is an adaptation of the classical Hill estimator [23] to our conditional framework. In the following, this estimator is referred to as the nearest neighbor Hill estimator. The asymptotic normality of $\hat{\gamma}_{n}^{\mathrm{H}}(t)$ is a direct consequence of Theorem 1 with $\mathcal{M S B}(1,1)=1$ and $\mathcal{A} \mathcal{V}(1,1)=1$. The associated conditional quantile estimator $\hat{q}^{\mathrm{H}}\left(\alpha_{n, t}, t\right)$ admits the same limiting distribution as in Theorem 2.

\subsection{Nearest neighbor Zipf estimator}

The Zipf estimator, initially introduced in the unconditional case [24, 28], can be adapted to our framework by remarking that the pairs

$$
\left(\log \left(\frac{m_{n, t}}{i}\right), \log \left(Z_{m_{n, t}-i+1, m_{n, t}}\right)\right), i=1, \ldots, k_{n, t},
$$

are approximatively distributed on a line of slope $\gamma(t)$. Then, a least-squares estimation yields the following estimator of $\gamma(t)$ :

$$
\sum_{i=1}^{k_{n, t}} \mu_{i, n, t} C_{i, n, t} / \sum_{i=1}^{k_{n, t}} \mu_{i, n, t}
$$

where

$$
\mu_{i, n, t}=\frac{1}{i} \sum_{j=1}^{i} \log \left(m_{n, t} / j\right)-\frac{1}{k_{n, t}} \sum_{j=1}^{k_{n, t}} \log \left(m_{n, t} / j\right) .
$$

Remarking that $\mu_{i, n, t}$ is asymptotically equivalent to $\log \left(k_{n, t} / i\right)$, the nearest neighbor Zipf estimator is defined as

$$
\hat{\gamma}_{n}^{\mathrm{Z}}(t)=\hat{\gamma}_{n}(t, 2,1)=\sum_{i=1}^{k_{n, t}} \log \left(k_{n, t} / i\right) C_{i, n, t} / \sum_{i=1}^{k_{n, t}} \log \left(k_{n, t} / i\right) .
$$

Theorem 1 holds for this estimator with $\mathcal{M S B}(2,1)=1 / 3$ and $\mathcal{A V}(2,1)=2$. Similarly, Theorem 2 also holds for the conditional quantile estimator $\hat{q}^{\mathrm{Z}}\left(\alpha_{n, t}, t\right)$ derived from the nearest neighbor Zipf estimator.

\subsection{Controlling the asymptotic mean-squared error}

Following Theorem 1 , the asymptotic mean-squared error of the estimator $\hat{\gamma}_{n}(t, a, \lambda)$ can be defined as

$$
\mathcal{A M S E}(a, \lambda)=\Delta^{2}\left(\frac{m_{n, t}}{k_{n, t}}, t\right) \mathcal{M S B}(a, \lambda)+\frac{\gamma^{2}(t) \mathcal{A} \mathcal{V}(a, \lambda)}{k_{n, t}},
$$


One way to choose the log-gamma parameters could be to find the ones minimizing the asymptotic mean-squared error. In practice, the function $\Delta$ is unknown and thus the asymptotic mean-squared error cannot be evaluated. To overcome this problem, it is possible to introduce an upper bound on $\mathcal{A M S E}(a, \lambda)$. Introducing $\pi(a, \lambda)=\mathcal{M S B}(a, \lambda) \mathcal{A V}(a, \lambda)$, we obtain for all $\lambda \in(0,1]$ and $a \in\left[1, a_{\max }\right]$,

$$
\begin{aligned}
\mathcal{A M S E}(a, \lambda) & =\frac{\pi(a, \lambda)}{k_{n, t}}\left\{\frac{\xi^{2}(t)+o(1)}{\mathcal{A V}(a, \lambda)}+\frac{\gamma^{2}(t)}{\mathcal{M S B}(a, \lambda)}\right\} \\
& \leq \frac{\pi(a, \lambda)}{k_{n, t}}\left\{\xi^{2}(t)+o(1)+\gamma^{2}(t)\left(2 a_{\max }-1\right)\right\} .
\end{aligned}
$$

We thus propose to consider the log-gamma parameters defined as:

$$
\left(a_{\pi}, \lambda_{\pi}\right)=\underset{a, \lambda}{\arg \min } \pi(a, \lambda),
$$

leading to the estimator $\hat{\gamma}_{n}^{\pi}(t, a, \lambda)=\hat{\gamma}_{n}\left(t, a_{\pi}, \lambda_{\pi}\right)$. Annulling the partial derivative of $\pi(a, \lambda)$ with respect to $\lambda$ yields $\lambda_{\pi}=4 /\left(1+2 a_{\pi}\right)$ whereas it is not possible to find an explicit value for $a_{\pi}$. A numerical optimization yields $a_{\pi} \approx 2.19$. Theorem 1 holds with $\mathcal{M S B}\left(a_{\pi}, \lambda_{\pi}\right) \approx 0.40$ and $\mathcal{A} \mathcal{V}\left(a_{\pi}, \lambda_{\pi}\right) \approx 1.51$, and Theorem 2 holds for the corresponding conditional quantile estimator $\hat{q}^{\pi}\left(\alpha_{n, t}, t\right)$.

\subsection{Discussion}

The three previously introduced log-gamma densities are represented on Figure 1. The nearest neighbor Hill estimator gives the same weight to all the $k_{n, t}$ largest observations. The nearest neighbor Zipf estimator corresponds to a decreasing log-gamma density. Finally, the log-gamma density used in $\hat{\gamma}_{n}^{\pi}($. has a mode in $(0,1)$. A heavy left tail for the log-gamma distribution (5) gives large weights to large observations in (4) and yields large asymptotic variances:

$$
\mathcal{A} \mathcal{V}(2,1)>\mathcal{A} \mathcal{V}\left(a_{\pi}, \lambda_{\pi}\right)>\mathcal{A} \mathcal{V}(1,1) .
$$

Asymptotic bias have an opposite behavior:

$$
\mathcal{M S B}(2,1)<\mathcal{M S B}\left(a_{\pi}, \lambda_{\pi}\right)<\mathcal{M S B}(1,1) .
$$

It is thus not possible to find log-gamma parameters giving rise to the best estimator both in terms of asymptotic bias and variance. However, for a given mean-squared bias, it is possible to compute the best asymptotic variance. Letting $\mathcal{M S B}(a, \lambda)=b$, we obtain $\lambda(a, b)=1 /(b(2 a-1))$ and consequently

$$
\mathcal{A} \mathcal{V}(a, \lambda(a, b))=b \frac{\Gamma(2 a)}{\Gamma^{2}(a)}\left\{2-\frac{1}{b(2 a-1)}\right\}^{1-2 a},
$$

where $a \geq \max \{1,(1+b) /(2 b)\}$ in order to ensure $0<\lambda(a, b) \leq 1$. The optimal asymptotic variance for a fixed mean-squared bias $b$ can be obtained by minimizing this quantity with respect to $a$ :

$$
\mathcal{O} \mathcal{A} \mathcal{V}(b)=\min _{a \geq \max \{1,(1+b) /(2 b)\}} \mathcal{A V}(a, \lambda(a, b)) .
$$


Here again, an explicit solution is not available. The graph of the function $\mathcal{O} \mathcal{A V}$ obtained by numerical optimization is depicted on Figure 2. Some level curves of $\pi(a, \lambda)$ are also represented. It appears that $\hat{\gamma}_{n}^{\pi}$ and $\hat{\gamma}_{n}^{\mathrm{H}}$ can be considered as optimal estimators since, for a fixed value of mean-squared bias, they have the optimal asymptotic variance. In contrast, the nearest neighbor Zipf estimator is not optimal. It is possible to build an estimator with same mean-squared bias $(=1 / 3)$ and smaller asymptotic variance $(\approx 1.85)$.

\section{An application to rainfall data}

Extreme rainfall statistics are often used when a flood occurred to assess the rarity of such an event. A typical question is to estimate what is the amount of rain on one hour that is exceeded once in 10 years. Mathematically speaking, the problem is to estimate the 10-years quantile of rainfall on one hour.

In [10], a Bayesian approach is used to model extreme precipitations in Front Range of Colorado. The excess distribution is represented by a Generalized Pareto Distribution whose parameters are stochastic processes depending on some geographical variables. An alternative approach consists in modelling the rainfall process itself by a max-stable process. We refer to $[5,27]$ for applications to the daily rainfalls in the Netherlands and in the USA respectively. Here, we consider hourly rainfall observations at 142 stations in the Cévennes-Vivarais region (southern part of France) during 7 years. In this context, the variable of interest $Y$ is the hourly rainfall and the covariate $x$ is the three dimensional geographical location ( $x_{1}$ is the longitude, $x_{2}$ is the latitude and $x_{3}$ is the altitude). The set of coordinates $S=\left\{\left(x_{1, j}, x_{2, j}, x_{3, j}\right), j=1, \ldots, 142\right\}$ of the raingauge stations is depicted on Figure 3 . The total number of observations is $n=264056$.

Let us first focus on the estimation of the conditional tail index $\gamma(x)$ as a function of $x$. To this aim, the three previously described estimators $\hat{\gamma}_{n}^{\mathrm{H}}, \hat{\gamma}_{n}^{\mathrm{Z}}$ and $\hat{\gamma}_{n}^{\pi}$ are used. All of them depend on the choice of $m_{n, t}$ and $k_{n, t}$. For the sake of simplicity, these parameters are chosen to be independent of the location $t$. They are selected by minimizing some dissimilarity measure between the estimators:

$$
(\hat{k}, \hat{m})=\underset{k_{n, t}, m_{n, t}}{\arg \min } \max _{t \in S} \mathcal{D}\left(\hat{\gamma}_{n}^{\mathrm{H}}(t), \hat{\gamma}_{n}^{\mathrm{Z}}(t), \hat{\gamma}_{n}^{\pi}(t)\right)
$$

with $\mathcal{D}\left(u_{1}, u_{2}, u_{3}\right) \stackrel{\text { def }}{=} \max \left\{\left|u_{1}-u_{2}\right|,\left|u_{2}-u_{3}\right|,\left|u_{3}-u_{1}\right|\right\}$. This heuristics is sometimes used in nonparametric estimation. It relies on the idea that, for a properly chosen pair $(\hat{k}, \hat{m})$, all three estimates should approximatively give the same tail index. This procedure yields $\hat{m} / n=55 \%$ and $\hat{k} / \hat{m}=5.5 \%$. Note that the graphical representation of the estimated tail index as a function of a three dimensional covariate is not possible. The role of the altitude $x_{3}$ is illustrated on Figure 4 while the role of the planar coordinates $\left(x_{1}, x_{2}\right)$ is represented on Figure 5. The shapes of the three curves representing the estimated tail index as a function of the altitude are qualitatively the same. The tail index is a decreasing function of the altitude till $x_{3}=800$ meters and is constant for altitudes ranging from 800 and 1600 meters. This phenomena can be interpreted since extreme hourly rainfalls are more likely to occur in the plains than in the mountains. This result is confirmed by Figure 5 where $\hat{\gamma}_{n}^{\pi}$ is represented 
as a function of the longitude and latitude. For the visualization sake, the pointwise estimations obtained on the set $S$ are interpolated by kriging. Here again, one can observe that heavy tails are obtained in the plains (Rhône Valley and Mediterranean coast). Similar results are obtained concerning the 10-years return level. It appears on Figure 6 that the considered return level is globally decreasing with the altitude. However, the observed variability indicates that altitude is not the unique factor. Indeed, one can see on Figure 7 that the Valence area of Rhône Valley does not suffer from high return levels whereas the southern part does.

The drift of the rainfall rate as a function of the altitude is in agreement with the rainfall descriptive statistics in the region [26]. Since in this region low altitude areas are flat areas and are closed to the sea, the deconvolution of physical processes involved in such an altitude-rainfall rate relationship is not trivial. Therefore, the enhancement of extreme rainfall rates could be either a regional specificity: it could be due the supply of warm and moist air by northward low level winds over the Mediterranean sea; or a more universal phenomena: flat areas are the more efficient in capturing the solar energy which is in turn available to involve deep convective clouds.

Finally, let us emphasize that these results are obtained under a temporal stationarity assumption of the rainfalls. Indeed, the short observation period ( 7 years) does not allow to discern any trend in the time series. However, it would be interesting to take into account seasonal effects. To this end, our further work will consist in splitting the data into homogeneous time periods. Such seasonal approaches have already been considered in Bayesian models $[7,8]$.

\section{Proofs}

Some preliminary results are given in paragraph 6.1. Their proofs are postponed to paragraph 6.3 while main results are proved in paragraph 6.2 . For the sake of simplicity, in the sequel, we note $k_{t}$ for $k_{n, t}, \Delta_{t}$ for $\Delta\left(m_{n, t} / k_{n, t}, t\right), \alpha_{t}$ for $\alpha_{n, t}$ and $m_{t}$ for $m_{n, t}$. Letting $J_{k_{t}}=\left\{1, \ldots, k_{t}\right\}$ and $J_{m_{t}}=\left\{1, \ldots, m_{t}\right\}$, we finally introduce

- $\left\{V_{i}, i \in J_{m_{t}}\right\}$ a set of independent standard uniform variables,

- $V_{1, m_{t}} \leq \ldots \leq V_{m_{t}, m_{t}}$ the associated order statistics,

- $\left\{F_{i}, i \in J_{k_{t}}\right\}$ a set of independent standard exponential random variables.

\subsection{Preliminary results}

The first lemma provides a representation in distribution of the logarithm of the observations whose covariate is in the neighborhood of $t$.

Lemma 1. Under (A.1) and (A.3), if $k_{t} / m_{t} \rightarrow 0$ and $k_{t}^{2} \omega_{n}\left(m_{t}^{-(1+\delta)}\right) \rightarrow 0$ for some $\delta>0$, then, there exists an event $\mathcal{A}_{n}$ with $\mathbb{P}\left(\mathcal{A}_{n}\right) \rightarrow 1$ as $n \rightarrow \infty$ such that $\left\{\left(\log Z_{m_{t}-i+1, m_{t}}, i \in J_{k_{t}}\right) \mid \mathcal{A}_{n}\right\}$ has the same distribution as

$$
\left\{\left(\log q\left(V_{i, m_{t}}, t\right)+O_{\mathrm{P}}\left(\omega_{n}\left(m_{t}^{-(1+\delta)}\right)\right), i \in J_{k_{t}}\right) \mid \mathcal{A}_{n}\right\} .
$$


In order to be self-contained, we quote a lemma proved in [1]. This result provides an exponential regression model for rescaled log-spacings.

Lemma 2. Suppose (A.1), (A.2) and (B) hold. Then, the random vector

$$
\left\{i \log \left(\frac{q\left(V_{i, m_{t}}, t\right)}{q\left(V_{i+1, m_{t}}, t\right)}\right), i \in J_{k_{t}}\right\}
$$

has the same distribution as

$$
\left\{\left(\gamma(t)+\Delta\left(\frac{i}{k_{t}+1}, t\right)^{-\rho(t)}\right) F_{i}+\beta_{i, n}(t)+o_{\mathrm{P}}\left(\Delta_{t}\right), i \in J_{k_{t}}\right\},
$$

where

$$
\sum_{j=1}^{k_{t}}\left(\frac{1}{j} \int_{0}^{j / k_{t}} u(\nu) d \nu\right) \beta_{i, n}(t)=o_{\mathrm{P}}\left(\Delta_{t}\right)
$$

for every function u defined on $(0,1)$ satisfying

$$
\left|k_{t} \int_{(j-1) / k_{t}}^{j / k_{t}} u(\nu) d \nu\right| \leq g\left(\frac{j}{k_{t}+1}\right)
$$

for some fixed positive continuous function $g(., t)$ defined on $(0,1)$ and satisfying

$$
\int_{0}^{1} \max (1, \log (1 / s)) g(s) d s<\infty .
$$

The following lemma states that $p\left(j / k_{t}, a, \lambda\right)$ can be rewritten as $\frac{1}{j} \int_{0}^{j / k_{t}} u(\nu) d \nu$ where $u$ is a function satisfying (9) and (10).

Lemma 3. Let $a \geq 1$ and $0<\lambda \leq 1$. There exists a function $u$ satisfying (9) and (10) such that for all $j \in J_{k_{t}}$,

$$
p\left(j / k_{t}, a, \lambda\right)=\frac{1}{j} \int_{0}^{j / k_{t}} u(\nu) d \nu .
$$

Finally, the following lemma is a simple unconditioning tool for determining the asymptotic distribution of a random variable.

Lemma 4. Let $\left(X_{n}\right)$ and $\left(Y_{n}\right)$ be two sequences of real random variables. Suppose there exists an event $\mathcal{A}_{n}$ such that $\left(X_{n} \mid \mathcal{A}_{n}\right) \stackrel{d}{=}\left(Y_{n} \mid \mathcal{A}_{n}\right)$ with $\mathbb{P}\left(\mathcal{A}_{n}\right) \rightarrow 1$. Then, $Y_{n} \stackrel{d}{\rightarrow} Y$ implies $X_{n} \stackrel{d}{\rightarrow} Y$.

\subsection{Proofs of main results}

The following result is a consequence of Lemmas 1-3. It establishes a representation of the log-spacings in terms of standard exponential random variables which is the cornerstone of the proof of Theorem 1. We refer to [15], Theorem 3.5.2, for the approximation of the nearest neighbors distribution using the Hellinger distance and to [16] for the study of their asymptotic distribution. 
Proposition 1. Suppose (A.1), (A.2), (A.3) and (B) hold. If, moreover, $k_{t}^{2} \omega_{n}\left(m_{t}^{-(1+\delta)}\right) \rightarrow 0$ for some $\delta>0$ then

$$
\left\{C_{i, n, t}, i \in J_{k_{t}} \mid \mathcal{A}_{n}\right\} \stackrel{d}{=}\left\{C_{i, n, t}^{(1)}+C_{i, n, t}^{(2)}, i \in J_{k_{t}} \mid \mathcal{A}_{n}\right\}
$$

where

$$
\begin{aligned}
\left\{C_{i, n, t}^{(1)}, i \in J_{k_{t}}\right\} & \stackrel{d}{=}\left\{\left(\gamma(t)+\Delta\left(\frac{i}{k_{t}+1}, t\right)^{-\rho(t)}\right) F_{i}+\beta_{i, n}(t)+o_{\mathrm{P}}\left(\Delta_{t}\right), i \in J_{k_{t}}\right\} \\
C_{i, n, t}^{(2)} & =O_{\mathrm{P}}\left(k_{t} \omega_{n}\left(m_{t}^{-(1+\delta)}\right)\right) \text { uniformly in } i \in J_{k_{t}} .
\end{aligned}
$$

and with

$$
\frac{1}{k_{t}} \sum_{i=1}^{k_{t}} p\left(i / k_{t}, a, \lambda\right) \beta_{i, n}(t)=o_{\mathrm{P}}\left(\Delta_{t}\right) .
$$

Proof of Proposition 1 - From Lemma 1,

$$
\begin{aligned}
\left\{C_{i, n, t}, i \in J_{k_{t}} \mid \mathcal{A}_{n}\right\} & \stackrel{d}{=}\left\{i \log \left(\frac{q\left(V_{i, m_{t}}, t\right)}{q\left(V_{i+1, m_{t}}, t\right)}\right)+i O_{\mathrm{P}}\left(\omega_{n}\left(m_{t}^{-(1+\delta)}\right)\right), i \in J_{k_{t}} \mid \mathcal{A}_{n}\right\} \\
& =\left\{C_{i, n, t}^{(1)}+C_{i, n, t}^{(2)}, i \in J_{k_{t}} \mid \mathcal{A}_{n}\right\},
\end{aligned}
$$

where

$$
C_{i, n, t}^{(1)}=i \log \left(\frac{q\left(V_{i, m_{t}}, t\right)}{q\left(V_{i+1, m_{t}}, t\right)}\right) \text { and } C_{i, n, t}^{(2)}=i O_{\mathrm{P}}\left(\omega_{n}\left(m_{t}^{-(1+\delta)}\right)\right) .
$$

From Lemmas 2 and $3,\left\{C_{i, n, t}^{(1)}, i \in J_{k_{t}}\right\}$ has the same distribution as

$$
\left\{\left(\gamma(t)+\Delta_{t}\left(\frac{i}{k_{t}+1}\right)^{-\rho(t)}\right) F_{i}+\beta_{i, n}(t)+o_{\mathrm{P}}\left(\Delta_{t}\right), i \in J_{k_{t}}\right\},
$$

with

$$
\frac{1}{k_{t}} \sum_{i=1}^{k_{t}} p\left(i / k_{t}, a, \lambda\right) \beta_{i, n}(t)=o_{\mathrm{P}}\left(\Delta_{t}\right) .
$$

Finally, for all $i \in J_{k_{t}}$,

$$
C_{i, n, t}^{(2)}=O_{\mathrm{P}}\left(k_{t} \omega_{n}\left(m_{t}^{-(1+\delta)}\right)\right),
$$

and the result is proved.

Proof of Theorem 1 - Let us consider the random variables defined as

$$
\begin{aligned}
\Lambda_{n}^{(1)} & =k_{t}^{1 / 2}\left\{\hat{\gamma}_{n}(t, a, \lambda)-\gamma(t)-\Delta_{t} \mathcal{A B}(a, \lambda, \rho(t))\right\} \\
\Lambda_{n}^{(2)} & =k_{t}^{1 / 2}\left(\frac{\sum_{i=1}^{k_{t}} p\left(i / k_{t}, a, \lambda\right)\left(C_{i, n, t}^{(1)}+C_{i, n, t}^{(2)}\right)}{\sum_{i=1}^{k_{t}} p\left(i / k_{t}, a, \lambda\right)}-\gamma(t)-\Delta_{t} \mathcal{A B}(a, \lambda, \rho(t))\right)
\end{aligned}
$$


Proposition 1 states that $\left\{\Lambda_{n}^{(1)} \mid \mathcal{A}_{n}\right\} \stackrel{d}{=}\left\{\Lambda_{n}^{(2)} \mid \mathcal{A}_{n}\right\}$. From Lemma 4, to prove Theorem 1 , it is sufficient to show that $\Lambda_{n}^{(2)}$ converges in distribution to a $\mathcal{N}\left(0, \gamma^{2}(t) \mathcal{A} \mathcal{V}(a, \lambda)\right)$ random variable. Introducing

$$
\begin{aligned}
T_{1, n} & =\sum_{i=1}^{k_{t}} p\left(i / k_{t}, a, \lambda\right)\left(F_{i}-1\right), & T_{2, n} & =\sum_{i=1}^{k_{t}} p\left(i / k_{t}, a, \lambda\right)\left(\frac{i}{k_{t}+1}\right)^{-\rho(t)}\left(F_{i}-1\right), \\
T_{3, n} & =\sum_{i=1}^{k_{t}} p\left(i / k_{t}, a, \lambda\right) \beta_{i, n}(t), & T_{4, n} & =\Delta_{t} \sum_{i=1}^{k_{t}} p\left(i / k_{t}, a, \lambda\right)\left(\frac{i}{k_{t}+1}\right)^{-\rho(t)}, \\
T_{5, n} & =\sum_{i=1}^{k_{t}} p\left(i / k_{t}, a, \lambda\right), & T_{6, n} & =\left(\sum_{i=1}^{k_{t}} p^{2}\left(i / k_{t}, a, \lambda\right)\right)^{1 / 2}
\end{aligned}
$$

Proposition 1 entails the following expansion:

$$
\begin{aligned}
\frac{T_{5, n}}{T_{6, n}}\left(\frac{\sum_{i=1}^{k_{t}} p\left(i / k_{t}, a, \lambda\right) C_{i, n, t}^{(1)}}{\sum_{i=1}^{k_{t}} p\left(i / k_{t}, a, \lambda\right)}-\gamma(t)-\frac{T_{4, n}}{T_{5, n}}\right) \stackrel{d}{=} \gamma(t) \frac{T_{1, n}}{T_{6, n}}+\Delta_{t} \frac{T_{2, n}}{T_{6, n}}+\frac{T_{3, n}}{T_{6, n}} \\
+\frac{T_{5, n}}{T_{6, n}} o_{\mathrm{P}}\left(\Delta_{t}\right) .
\end{aligned}
$$

From Lindeberg theorem, a sufficient condition for $T_{1, n} / T_{6, n} \stackrel{d}{\rightarrow} \mathcal{N}(0,1)$ is

$$
\sum_{i=1}^{k_{t}} p^{3}\left(i / k_{t}, a, \lambda\right) / T_{6, n}^{3} \rightarrow 0 .
$$

Since for any integrable function $\varphi$, the following convergence of Riemann sum holds,

$$
\frac{1}{k_{t}} \sum_{i=1}^{k_{t}} \varphi\left(\frac{i}{k_{t}}\right) \rightarrow \int_{0}^{1} \varphi(s) d s
$$

it follows that $T_{6, n}=k_{t}^{1 / 2} \mathcal{A} \mathcal{V}(a, \lambda)^{1 / 2}(1+o(1))$. Thus, using (14), we have

$$
\frac{1}{k_{t}} \sum_{i=1}^{k_{t}} p^{3}\left(i / k_{t}, a, \lambda\right)=\frac{\Gamma(3 a)}{\Gamma^{3}(a)}(3-2 \lambda)^{-3 a}(1+o(1)),
$$

and therefore

$$
\sum_{i=1}^{k_{t}} p^{3}\left(i / k_{t}, a, \lambda\right) / T_{6, n}^{3}=O\left(k_{t}^{-1 / 2}\right),
$$

showing that condition (13) is satisfied and

$$
T_{1, n} / T_{6, n} \stackrel{d}{\rightarrow} \mathcal{N}(0,1) .
$$

Next, let us focus on $T_{2, n} / T_{6, n}$. Remarking that this term is centered with finite variance, it follows that

$$
T_{2, n} / T_{6, n}=O_{\mathrm{P}}(1) .
$$

Equation (11) in Proposition 1 yields

$$
T_{3, n} / T_{6, n}=o_{\mathrm{P}}\left(k_{t}^{1 / 2} \Delta_{t}\right)=o_{\mathrm{P}}(1) .
$$


Finally, from (14) we obtain,

$$
\begin{aligned}
& T_{4, n} / T_{5, n}=\Delta_{t} \mathcal{A} \mathcal{B}(a, \lambda, \rho(t))(1+o(1)), \\
& T_{5, n} / T_{6, n}=k_{t}^{1 / 2} \mathcal{A} \mathcal{V}(a, \lambda)^{-1 / 2}(1+o(1)) .
\end{aligned}
$$

Replacing (15)-(19) in (12) shows that

$$
k_{t}^{1 / 2}\left(\frac{\sum_{i=1}^{k_{t}} p\left(i / k_{t}, a, \lambda\right) C_{i, n, t}^{(1)}}{\sum_{i=1}^{k_{t}} p\left(i / k_{t}, a, \lambda\right)}-\gamma(t)-\Delta_{t} \mathcal{A} \mathcal{B}(a, \lambda, \rho(t))\right)
$$

converges in distribution to a $\mathcal{N}\left(0, \gamma^{2}(t) \mathcal{A} \mathcal{V}(a, \lambda)\right)$ random variable. Taking account of

$$
k_{t}^{1 / 2} \frac{\sum_{i=1}^{k_{t}} p\left(i / k_{t}, a, \lambda\right) C_{i, n, t}^{(2)}}{\sum_{i=1}^{k_{t}} p\left(i / k_{t}, a, \lambda\right)}=O_{\mathrm{P}}\left(k_{t}^{3 / 2} \omega_{n}\left(m_{t}^{-(1+\delta)}\right)\right)=o_{\mathrm{P}}(1)
$$

concludes the proof.

Proof of Theorem 2 - Observing that

$$
\log \hat{q}\left(\alpha_{t}, t\right)=\log Z_{m_{t}-k_{t}+1, m_{t}}+\hat{\gamma}_{n}(t, a, \lambda) \log \left(\frac{k_{t}}{m_{t} \alpha_{t}}\right),
$$

leads to the following expansion:

$$
\begin{aligned}
\log \left(\frac{\hat{q}\left(\alpha_{t}, t\right)}{q\left(\alpha_{t}, t\right)}\right) & -\log \left(\frac{k_{t}}{m_{t} \alpha_{t}}\right) \Delta_{t} \mathcal{A B}(a, \lambda, \rho(t)) \\
& =\log \left(\frac{Z_{m_{t}-k_{t}+1, m_{t}}}{q\left(k_{t} / m_{t}, t\right)}\right) \\
& +\left\{\hat{\gamma}_{n}(t, a, \lambda)-\gamma(t)-\Delta_{t} \mathcal{A B}(a, \lambda, \rho(t))\right\} \log \left(\frac{k_{t}}{m_{t} \alpha_{t}}\right) \\
& -\left\{\log \left(\frac{q\left(\alpha_{t}, t\right)}{q\left(k_{t} / m_{t}, t\right)}\right)-\gamma(t) \log \left(\frac{k_{t}}{m_{t} \alpha_{t}}\right)\right\} \\
& =\zeta_{1, n}+\zeta_{2, n}+\zeta_{3, n} .
\end{aligned}
$$

First, from Lemma 1,

$$
\zeta_{1, n} \stackrel{d}{=} \log \left(\frac{q\left(V_{k_{t}, m_{t}}, t\right)}{q\left(k_{t} / m_{t}, t\right)}\right)+O_{\mathrm{P}}\left(\omega_{n}\left(m_{t}^{-(1+\delta)}\right)\right),
$$

and assumptions (A.1), (A.3) imply that

$$
\left|\log \left(\frac{q\left(V_{k_{t}, m_{t}}, t\right)}{q\left(k_{t} / m_{t}, t\right)}\right)\right|=O_{\mathrm{P}}\left(\Delta_{t}\right)\left|\log \left(\frac{m_{t}}{k_{t}} V_{k_{t}, m_{t}}\right)\right| .
$$

Moreover, under (B), it is well-known that

$$
k_{t}^{1 / 2}\left(\frac{m_{t}}{k_{t}} V_{k_{t}, m_{t}}-1\right) \stackrel{d}{\rightarrow} \mathcal{N}(0,1),
$$


(see for instance [19]) and thus

$$
\zeta_{1, n}=O_{\mathrm{P}}\left(\Delta_{t} k_{t}^{-1 / 2}\right)+O_{\mathrm{P}}\left(\omega_{n}\left(m_{t}^{-(1+\delta)}\right)\right) .
$$

Besides, from Theorem 1, we have

$$
k_{t}^{1 / 2} / \log \left(k_{t} /\left(m_{t} \alpha_{t}\right)\right) \zeta_{2, n} \stackrel{d}{\rightarrow} \mathcal{N}\left(0, \gamma^{2}(t) \mathcal{A} \mathcal{V}(a, \lambda)\right),
$$

and, finally, (7) entails that

$$
\log \left(\frac{q\left(\alpha_{t}, t\right)}{q\left(k_{t} / m_{t}, t\right)}\right)=\gamma(t) \log \left(\frac{k_{t}}{m_{t} \alpha_{t}}\right)+\int_{m_{t} / k_{t}}^{\alpha_{t}^{-1}} \frac{\Delta(u, t)}{u} d u .
$$

Since $\alpha_{t}<k_{t} / m_{t},(\mathbf{A} .3)$ yields

$$
\left|\zeta_{3, n}\right| \leq \int_{m_{t} / k_{t}}^{\alpha_{t}^{-1}} \frac{|\Delta(u, t)|}{u} d u \leq \Delta_{t} \log \left(\frac{k_{t}}{m_{t} \alpha_{t}}\right)
$$

and collecting (20)-(22) concludes the proof.

\subsection{Proofs of auxiliary results}

Proof of Lemma 1 - Under (A1) the function $q(., t)$ is continuous. Since the random variables $\left\{Z_{i}, i \in J_{m_{t}}\right\}$ are independent, we have:

$$
\left\{\log Z_{i}, i \in J_{m_{t}}\right\} \stackrel{d}{=}\left\{\log q\left(V_{i}, x_{i}^{*}\right) i=1 \in J_{m_{t}}\right\},
$$

where $x_{i}^{*}$ is the covariate associated to $Z_{i}$. Denoting by $\psi(i)$ the random index of the covariate associated to the observation $Z_{m_{t}-i+1, m_{t}}$, we obtain

$$
\left\{\log Z_{m_{t}-i+1, m_{t}}, i \in J_{m_{t}}\right\} \stackrel{d}{=}\left\{\log q\left(V_{\psi(i)}, x_{\psi(i)}^{*}\right) i \in J_{m_{t}}\right\} .
$$

Let us consider the event $\mathcal{A}_{n}=\mathcal{A}_{1, n} \cap \mathcal{A}_{2, n}$ where

$$
\begin{aligned}
& \mathcal{A}_{1, n}=\left\{\min _{i \in J_{k_{t}} \backslash\left\{k_{t}\right\}} \log \left(\frac{q\left(V_{i, m_{t}}, u_{i}\right)}{q\left(V_{i+1, m_{t}}, u_{i+1}\right)}\right)>0, \forall\left(u_{1}, \ldots, u_{k_{t}}\right) \in \mathcal{V}_{n, t}\right\} \text { and } \\
& \mathcal{A}_{2, n}=\left\{\min _{i \in J_{m_{t}} \backslash J_{k_{t}}} \log \left(\frac{q\left(V_{k_{t}, m_{t}}, u_{k_{t}}\right)}{q\left(V_{i, m_{t}}, u_{i}\right)}\right)>0, \forall\left(u_{k_{t}+1}, \ldots, u_{m_{t}}\right) \in \mathcal{V}_{n, t}\right\} .
\end{aligned}
$$

Conditionally to $\mathcal{A}_{1, n}$, the random variables $q\left(V_{i, m_{t}}, u_{i}\right), i \in J_{k_{t}}$ are ordered as

$$
q\left(V_{k_{t}, m_{t}}, u_{k_{t}}\right) \leq q\left(V_{k_{t}-1, m_{t}}, u_{k_{t}-1}\right) \leq \cdots \leq q\left(V_{1, m_{t}}, u_{1}\right),
$$

and, conditionally to $\mathcal{A}_{2, n}$, the remaining random variables $q\left(V_{i, m_{t}}, u_{i}\right), i \in$ $J_{m_{t}} \backslash J_{k_{t}}$ are smaller since

$$
\max _{i \in J_{m_{t}} \backslash J_{k_{t}}} q\left(V_{i, m_{t}}, u_{i}\right) \leq q\left(V_{k_{t}, m_{t}}, u_{k_{t}}\right) .
$$

Thus, conditionally to $\mathcal{A}_{n}$, the $k_{t}$ largest random values taken from the set $\left\{\log q\left(V_{\psi(i)}, x_{\psi(i)}^{*}\right), i \in J_{m_{t}}\right\}$ are $\left\{\log q\left(V_{i, m_{t}}, x_{\psi(i)}^{*}\right), i \in J_{k_{t}}\right\}$. Consequently, letting $T_{i} \stackrel{\text { def }}{=} x_{\psi(i)}^{*}$, we have:

$$
\left\{\log Z_{m_{t}-i+1, m_{t}}, i \in J_{k_{t}} \mid \mathcal{A}_{n}\right\} \stackrel{d}{=}\left\{\log q\left(V_{i, m_{t}}, T_{i}\right), i \in J_{k_{t}} \mid \mathcal{A}_{n}\right\} .
$$


To conclude the proof, it remains to show that

$$
\log \left(\frac{q\left(V_{i, m_{t}}, T_{i}\right)}{q\left(V_{i, m_{t}}, t\right)}\right)=O_{\mathrm{P}}\left(\omega_{n}\left(m_{t}^{-(1+\delta)}\right)\right),
$$

uniformly in $i \in J_{k_{t}}$ and that

$$
\mathbb{P}\left(\mathcal{A}_{n}\right) \rightarrow 1
$$

as $n \rightarrow \infty$. Let us define $\delta_{m_{t}}=m_{t}^{-(1+\delta)}$ and consider the event

$$
\mathcal{A}_{3, n}=\left\{V_{1, m_{t}}>\delta_{m_{t}}\right\} \cap\left\{V_{m_{t}, m_{t}}<1-\delta_{m_{t}}\right\}
$$

Under $\mathcal{A}_{3, n}$, we have $\delta_{m_{t}}<V_{i, m_{t}}<1-\delta_{m_{t}}$ for all $i \in J_{m_{t}}$. Hence,

$$
\begin{aligned}
& \mathbb{P}\left(\left|\log \left(\frac{q\left(V_{i, m_{t}}, T_{i}\right)}{q\left(V_{i, m_{t}}, t\right)}\right)\right|>\omega_{n}\left(m_{t}^{-(1+\delta)}\right)\right) \\
\leq & \mathbb{P}\left(\left|\log \left(\frac{q\left(V_{i, m_{t}}, T_{i}\right)}{q\left(V_{i, m_{t}}, t\right)}\right)\right|>\omega_{n}\left(m_{t}^{-(1+\delta)}\right) \mid \mathcal{A}_{3, n}\right) \mathbb{P}\left(\mathcal{A}_{3, n}\right)+\mathbb{P}\left(\mathcal{A}_{3, n}^{C}\right),
\end{aligned}
$$

where $\mathcal{A}_{3, n}^{C}$ is the complementary event associated to $\mathcal{A}_{3, n}$. Since under $\mathcal{A}_{3, n}$,

$$
\left|\log \left(\frac{q\left(V_{i, m_{t}}, T_{i}\right)}{q\left(V_{i, m_{t}}, t\right)}\right)\right| \leq \omega_{n}\left(m_{t}^{-(1+\delta)}\right),
$$

for all $i \in J_{k_{t}}$, it is clear that

$$
\mathbb{P}\left(\left|\log \left(\frac{q\left(V_{i, m_{t}}, T_{i}\right)}{q\left(V_{i, m_{t}}, t\right)}\right)\right|>\omega_{n}\left(m_{t}^{-(1+\delta)}\right) \mid \mathcal{A}_{3, n}\right)=0 .
$$

Remarking that

$\mathbb{P}\left(\mathcal{A}_{3, n}\right) \geq \mathbb{P}\left(V_{1, m_{t}}>\delta_{m_{t}}\right)+\mathbb{P}\left(V_{m_{t}, m_{t}}<1-\delta_{m_{t}}\right)-1=2 \mathbb{P}\left(V_{1, m_{t}}>\delta_{m_{t}}\right)-1 \rightarrow 1$, since $V_{m_{t}, m_{t}} \stackrel{d}{=} 1-V_{1, m_{t}}$ and $\mathbb{P}\left(V_{1, m_{t}}>\delta_{m_{t}}\right)=\left(1-\delta_{m_{t}}\right)^{m_{t}} \rightarrow 1$ concludes the proof of (23). Furthermore, for all $\left(u_{i}, u_{j}\right) \in \mathcal{V}_{n, t}^{2}$, we have, on the one hand

$$
\begin{aligned}
\log \left(\frac{q\left(V_{j, m_{t}}, u_{j}\right)}{q\left(V_{i, m_{t}}, u_{i}\right)}\right) & =\log \left(\frac{q\left(V_{j, m_{t}}, t\right)}{q\left(V_{i, m_{t}}, t\right)}\right)+\log \left(\frac{q\left(V_{j, m_{t}}, u_{j}\right)}{q\left(V_{j, m_{t}}, t\right)}\right)+\log \left(\frac{q\left(V_{i, m_{t}}, t\right)}{q\left(V_{i, m_{t}}, u_{i}\right)}\right) \\
& \geq \log \left(\frac{q\left(V_{j, m_{t}}, t\right)}{q\left(V_{i, m_{t}}, t\right)}\right)-2 \omega_{n}\left(\delta_{m_{t}}\right),
\end{aligned}
$$

and on the other hand,

$$
\begin{aligned}
\min _{i \in J_{m_{t}} \backslash J_{k_{t}}} \log \left(\frac{q\left(V_{k_{t}, m_{t}}, u_{k_{t}}\right)}{q\left(V_{i, m_{t}}, u_{i}\right)}\right) & \geq \min _{i \in J_{m_{t}} \backslash J_{k_{t}}} \log \left(\frac{q\left(V_{k_{t}, m_{t}}, t\right)}{q\left(V_{i, m_{t}}, t\right)}\right)-2 \omega_{n}\left(\delta_{m_{t}}\right) \\
& \geq \log \left(\frac{q\left(V_{k_{t}, m_{t}}, t\right)}{q\left(V_{k_{t}+1, m_{t}}, t\right)}\right)-2 \omega_{n}\left(\delta_{m_{t}}\right) .
\end{aligned}
$$

Consequently, considering the event

$$
\mathcal{A}_{4, n}=\left\{\min _{i \in J_{k_{t}}} \log \left(\frac{q\left(V_{i, m_{t}}, t\right)}{q\left(V_{i+1, m_{t}}, t\right)}\right)>2 \omega_{n}\left(\delta_{m_{t}}\right)\right\}
$$


it is clear that $\mathcal{A}_{3, n} \cap \mathcal{A}_{4, n} \subset \mathcal{A}_{n}$. It thus remains to prove that $\mathbb{P}\left(\mathcal{A}_{4, n}\right) \rightarrow 1$ to show (24). From (A.1), for all $\alpha \in(0,1)$,

$$
q(\alpha, t)=c(t) \exp \left\{\int_{1}^{\alpha^{-1}} \frac{\gamma(t)+\Delta(u, t)}{u} d u\right\} .
$$

Hence, for all $i \in J_{k_{t}}$,

$$
\log \left(\frac{q\left(V_{i, m_{t}}, t\right)}{q\left(V_{i+1, m_{t}}, t\right)}\right)=\int_{V_{i+1, m_{t}}^{-1}}^{V_{i, m_{t}}^{-1}} \frac{\gamma(t)+\Delta(u, t)}{u} d u .
$$

Since $V_{1, m_{t}}^{-1} \geq \ldots \geq V_{k_{t}+1, m_{t}}^{-1}=\left(m_{t} / k_{t}\right)\left(1+o_{\mathrm{P}}(1)\right) \stackrel{\mathrm{P}}{\rightarrow} \infty$, it follows from (A.3) that

$$
\log \left(\frac{q\left(V_{i, m_{t}}, t\right)}{q\left(V_{i+1, m_{t}}, t\right)}\right) \geq\left(\gamma(t)-\left|\Delta\left(V_{k_{t}+1, m_{t}}^{-1}, t\right)\right|\right) \log \left(\frac{V_{i+1, m_{t}}}{V_{i, m_{t}}}\right),
$$

leading to

$$
\begin{aligned}
\mathbb{P}\left(\mathcal{A}_{4, n}\right) & \geq \mathbb{P}\left(\left(\gamma(t)-\left|\Delta\left(V_{k_{t}+1, m_{t}}^{-1}, t\right)\right|\right) \min _{i \in J_{k_{t}}} \log \left(\frac{V_{i+1, m_{t}}}{V_{i, m_{t}}}\right)>2 \omega_{n}\left(\delta_{m_{t}}\right)\right) \\
& \geq \mathbb{P}\left(\left\{\min _{i \in J_{k_{t}}} \log \left(\frac{V_{i+1, m_{t}}}{V_{i, m_{t}}}\right) \geq \frac{4 \omega_{n}\left(\delta_{m_{t}}\right)}{\gamma(t)}\right\} \cap\left\{\left|\Delta\left(V_{k_{t}+1, m_{t}}^{-1}, t\right)\right|<\gamma(t) / 2\right\}\right) \\
& \geq \mathbb{P}\left(\min _{i \in J_{k_{t}}} \log \left(\frac{V_{i+1, m_{t}}}{V_{i, m_{t}}}\right) \geq \frac{4 \omega_{n}\left(\delta_{m_{t}}\right)}{\gamma(t)}\right)+\mathbb{P}\left(\left|\Delta\left(V_{k_{t}+1, m_{t}}^{-1}, t\right)\right|<\gamma(t) / 2\right)-1 \\
& \stackrel{\text { def }}{=} P_{1, m_{t}}+P_{2, m_{t}}-1 .
\end{aligned}
$$

In view of Rényi representation for uniform ordered random variables,

$$
\left\{i \log \left(V_{i, m_{t}}^{-1} / V_{i+1, m_{t}}^{-1}\right), i \in J_{k_{t}}\right\} \stackrel{d}{=}\left\{F_{i}, i \in J_{k_{t}}\right\},
$$

we have

$$
\begin{aligned}
P_{1, m_{t}} & =\mathbb{P}\left(\min _{i \in J_{k_{t}}} \frac{F_{i}}{i} \geq \frac{4 \omega_{n}\left(\delta_{m_{t}}\right)}{\gamma(t)}\right)=\prod_{i=1}^{k_{t}} \exp \left(-\frac{4 i \omega_{n}\left(\delta_{m_{t}}\right)}{\gamma(t)}\right) \\
& =\exp \left(-\frac{2}{\gamma(t)} k_{t}\left(k_{t}+1\right) \omega_{n}\left(\delta_{m_{t}}\right)\right) \rightarrow 1,
\end{aligned}
$$

since $k_{t}^{2} \omega_{n}\left(\delta_{m_{t}}\right) \rightarrow 0$. Furthermore, $V_{k_{t}+1, m_{t}} \stackrel{\mathrm{P}}{\rightarrow} 0$ and $\Delta\left(\alpha^{-1}, t\right) \rightarrow 0$ as $\alpha \rightarrow 0$ entail $P_{2, m_{t}} \rightarrow 1$. The conclusion follows.

Proof of Lemma 3 - Letting

$$
u(s)=\frac{d}{d s}(s p(s, a, \lambda))=\frac{\lambda^{-a}}{\Gamma(a)} s^{1 / \lambda-1}\left(\frac{(-\log (s))^{a-1}}{\lambda}-(a-1)(-\log (s))^{a-2}\right),
$$

it is easily seen that

$$
p\left(j / k_{t}, a, \lambda\right)=\frac{1}{j} \int_{0}^{j / k_{t}} u(\nu) d \nu
$$


Note that, if $a=1$, then $|u(s)|$ is a bounded function and thus it is easy to verify that (9) and (10) are satisfied. Let us now consider the case $a \neq 1$. Introducing $\tau=(2-a) \mathbb{I}\{a \in(1,2]\}$, we have:

$$
\begin{aligned}
|u(s)| & \leq \frac{\lambda^{-a}}{\Gamma(a)}(-\log (s))^{-\tau}\left(\frac{(-\log (s))^{a-1+\tau}}{\lambda}+(a-1)(-\log (s))^{a-2+\tau}\right) \\
& \leq \tilde{g}(s) \stackrel{\text { def }}{=} \begin{cases}\lambda^{-a} / \Gamma(a)\left(\lambda^{-1}+a-1\right)(-\log (s))^{a-1} & \text { if } s \in\left[0, \mathrm{e}^{-1}\right], \\
\lambda^{-a} / \Gamma(a)\left(\lambda^{-1}+a-1\right)(-\log (s))^{-\tau} & \text { if } s \in\left[\mathrm{e}^{-1}, 1\right] .\end{cases}
\end{aligned}
$$

Three situations are considered:

Situation a) - If $j<k_{t} \mathrm{e}^{-1}$, we have:

$\left|k_{t} \int_{(j-1) / k_{t}}^{j / k_{t}} u(\nu) d \nu\right| \leq \begin{cases}\lambda^{-a} / \Gamma(a)\left(\lambda^{-1}+a-1\right)\left(\log \left(k_{t} /(j-1)\right)\right)^{a-1} & \text { for } j \neq 1, \\ \lambda^{-a} / \Gamma(a)\left(\log \left(k_{t}\right)\right)^{a-1} & \text { for } j=1 .\end{cases}$

Besides, straightforward calculations lead to

$$
\left(\log \left(\frac{k_{t}}{j-1}\right)\right)^{a-1} \leq 2^{a-1}\left(\log \left(\frac{k_{t}+1}{j}\right)\right)^{a-1} \text { and }\left(\log \left(k_{t}\right)\right)^{a-1}<\left(\log \left(k_{t}+1\right)\right)^{a-1} .
$$

Hence,

$$
\left|k \int_{(j-1) / k_{t}}^{j / k_{t}} u(\nu) d \nu\right| \leq c_{1}(a, \lambda) \tilde{g}\left(\frac{j}{k_{t}+1}\right),
$$

where $c_{1}(a, \lambda)$ is a positive constant.

Situation b) - If $j>\left(k_{t}+1\right) \mathrm{e}^{-1}$, then

$$
\left|k_{t} \int_{(j-1) / k_{t}}^{j / k_{t}} u(\nu) d \nu\right| \leq \begin{cases}\frac{\lambda^{-a}}{\Gamma(a)}\left(\frac{1}{\lambda}+a-1\right)\left(\log \left(\frac{k_{t}}{j}\right)\right)^{-\tau} & \text { for } j \neq k_{t}, \\ \frac{\lambda^{-a}}{\Gamma(a)}\left(k_{t}-1\right)\left(\log \left(\frac{k_{t}}{k_{t}-1}\right)\right)^{a-1+\tau}\left(\log \left(\frac{k_{t}}{k_{t}-1}\right)\right)^{-\tau} & \text { for } j=k_{t} .\end{cases}
$$

Since $\log \left(k_{t} /\left(k_{t}-1\right)\right)<1 /\left(k_{t}-1\right)$ and $a-2+\tau \geq 0$, it follows that

$$
\left(k_{t}-1\right)\left(\log \left(\frac{k_{t}}{k_{t}-1}\right)\right)^{a-1+\tau}<\left(\frac{1}{k_{t}-1}\right)^{a-2+\tau} \leq 1,
$$

and thus

$$
\left|k_{t} \int_{(j-1) / k_{t}}^{j / k_{t}} u(\nu) d \nu\right| \leq \begin{cases}\lambda^{-a} / \Gamma(a)\left(\lambda^{-1}+a-1\right)\left(\log \left(k_{t} / j\right)\right)^{-\tau} & \text { for } j \neq k_{t}, \\ \lambda^{-a} / \Gamma(a)\left(\log \left(k_{t} /\left(k_{t}-1\right)\right)\right)^{-\tau} & \text { for } j=k_{t} .\end{cases}
$$

Remarking that

$$
\left(\log \left(\frac{k_{t}}{j}\right)\right)^{-\tau} \leq 3^{\tau}\left(\log \left(\frac{k_{t}+1}{j}\right)\right)^{-\tau} \text { and }\left(\log \left(\frac{k_{t}}{k_{t}-1}\right)\right)^{-\tau}<\left(\log \left(\frac{k_{t}+1}{k_{t}}\right)\right)^{-\tau},
$$

we have

$$
\left|k \int_{(j-1) / k_{t}}^{j / k_{t}} u(\nu) d \nu\right| \leq c_{2}(a, \lambda) \tilde{g}\left(\frac{j}{k_{t}+1}\right)
$$


where $c_{2}(a, \lambda)$ is a positive constant.

Situation c) - If $k_{t} \mathrm{e}^{-1}<j<\left(k_{t}+1\right) \mathrm{e}^{-1}$, then

$$
\left|k \int_{(j-1) / k_{t}}^{j / k_{t}} u(\nu) d \nu\right| \leq \max \left(\tilde{g}\left(\frac{j}{k_{t}}\right) ; \tilde{g}\left(\frac{j-1}{k_{t}}\right)\right)
$$

and one can show that

$$
\max \left(\tilde{g}\left(\frac{j}{k_{t}}\right) ; \tilde{g}\left(\frac{j-1}{k_{t}}\right)\right) \leq c_{3}(a, \lambda) \tilde{g}\left(\frac{j}{k_{t}+1}\right),
$$

where $c_{3}(a, \lambda)$ is a positive constant.

As a conclusion, for all $j \in J_{k_{t}},(9)$ is satisfied with

$$
g(s)= \begin{cases}c_{4}(a, \lambda)(-\log (s))^{a-1} & \text { if } s \in\left[0, \mathrm{e}^{-1}\right] \\ c_{4}(a, \lambda)(-\log (s))^{-\tau} & \text { if } s \in\left[\mathrm{e}^{-1}, 1\right]\end{cases}
$$

where $c_{4}(a, \lambda)=\lambda^{-a} / \Gamma(a)\left(\lambda^{-1}+a-1\right) \max \left(c_{1}(a, \lambda) ; c_{2}(a, \lambda) ; c_{3}(a, \lambda)\right)$. Finally, we have:

$$
\begin{aligned}
\int_{0}^{1} \max (1,-\log (s)) g(s) d s & =c_{4}(a, \lambda)\left\{\int_{0}^{\mathrm{e}^{-1}}(-\log (s))^{a-1} d s\right. \\
& \left.+\int_{\mathrm{e}^{-1}}^{1}(-\log (s))^{-\tau} d s\right\} \\
& \leq c_{4}(a, \lambda)(\Gamma(a+1)+\Gamma(1-\tau))<\infty
\end{aligned}
$$

since $\tau<1$, and (10) is proved.

Proof of Lemma 4 - For all $x \in \mathbb{R}$, the well-known expansion

$$
\mathbb{P}\left(X_{n} \leq x\right)=\mathbb{P}\left(\left\{X_{n} \leq x\right\} \mid \mathcal{A}_{n}\right) \mathbb{P}\left(\mathcal{A}_{n}\right)+\mathbb{P}\left(\left\{X_{n} \leq x\right\} \mid \mathcal{A}_{n}^{C}\right) \mathbb{P}\left(\mathcal{A}_{n}^{C}\right)
$$

leads to the following inequalities:

$$
\mathbb{P}\left(\left\{X_{n} \leq x\right\} \mid \mathcal{A}_{n}\right) \mathbb{P}\left(\mathcal{A}_{n}\right) \leq \mathbb{P}\left(X_{n} \leq x\right) \leq \mathbb{P}\left(\left\{X_{n} \leq x\right\} \mid \mathcal{A}_{n}\right) \mathbb{P}\left(\mathcal{A}_{n}\right)+\mathbb{P}\left(\mathcal{A}_{n}^{C}\right)
$$

Since $\left(X_{n} \mid \mathcal{A}_{n}\right) \stackrel{d}{=}\left(Y_{n} \mid \mathcal{A}_{n}\right)$, it follows that:

$$
\mathbb{P}\left(\left\{Y_{n} \leq x\right\} \cap \mathcal{A}_{n}\right) \leq \mathbb{P}\left(X_{n} \leq x\right) \leq \mathbb{P}\left(\left\{Y_{n} \leq x\right\} \cap \mathcal{A}_{n}\right)+\mathbb{P}\left(\mathcal{A}_{n}^{C}\right)
$$

Taking into account of

$$
\mathbb{P}\left(Y_{n} \leq x\right)-\mathbb{P}\left(\mathcal{A}_{n}^{C}\right) \leq \mathbb{P}\left(\left\{Y_{n} \leq x\right\} \cap \mathcal{A}_{n}\right) \leq \mathbb{P}\left(Y_{n} \leq x\right)
$$

leads to:

$$
\mathbb{P}\left(Y_{n} \leq x\right)-\mathbb{P}\left(\mathcal{A}_{n}^{C}\right) \leq \mathbb{P}\left(X_{n} \leq x\right) \leq \mathbb{P}\left(Y_{n} \leq x\right)+\mathbb{P}\left(\mathcal{A}_{n}^{C}\right)
$$

The conclusion is then straightforward since $\mathbb{P}\left(Y_{n} \leq x\right) \rightarrow \mathbb{P}(Y \leq x)$ and $\mathbb{P}\left(\mathcal{A}_{n}^{C}\right) \rightarrow 0$. 


\section{References}

[1] Beirlant, J., Dierckx, G., Guillou, A. and Stărică, C. (2002). On exponential representations of log-spacings of extreme order statistics. Extremes, 5, $157-180$.

[2] Beirlant, J. and Goegebeur, Y. (2003). Regression with response distributions of Pareto-type. Computational Statistics and Data Analysis, 42, 595-619.

[3] Beirlant, J. and Goegebeur, Y. (2004). Local polynomial maximum likelihood estimation for Pareto-type distributions. Journal of Multivariate Analysis, 89, 97-118.

[4] Bingham, N.H., Goldie, C.M. and Teugels, J.L. (1987). Regular variation. Encyclopedia of Mathematics and its Applications, 27, Cambridge University Press.

[5] Buishand, T.A., de Haan, L. and Zhou, C. (2008). On spatial extremes: with application to a rainfall problem. Annals of applied statistics, 2, 624642 .

[6] Chavez-Demoulin, V. and Davison, A.C. (2005). Generalized additive modelling of sample extremes. Journal of the Royal Statistical Society, series C., 54, 207-222.

[7] Coles, S. and Pericchi, L.R. (2003). Anticipating catastrophes through extreme value modelling. Applied Statistics, 52, 405-416.

[8] Coles, S., Pericchi, L.R. and Sisson, S. (2003). A fully probabilistic approach to extreme rainfall modeling. Journal of Hydrology, 273, 35-50.

[9] Consul, P.C. and Jain, G.C. (1971). On the log-gamma distribution and its properties. Statistische Hefe, 12(2), 100-106.

[10] Cooley, D., Nychka, D. and Naveau, P. (2007). Bayesian spatial modeling of extreme precipitation return levels. Journal of the American Statistical Association, 102, 824-840.

[11] Davison, A.C. and Ramesh, N.I. (2000). Local likelihood smoothing of sample extremes. Journal of the Royal Statistical Society, series B, 62, 191-208.

[12] Davison, A.C. and Smith, R.L. (1990). Models for exceedances over high thresholds. Journal of the Royal Statistical Society, series B, 52, 393-442.

[13] Dekkers, A. and de Haan, L. (1989). On the estimation of the extreme-value index and large quantile estimation. Annals of Statistics, 17, 1795-1832.

[14] Diebolt, J., Gardes, L., Girard, S. and Guillou, A. (2008). Bias-reduced extreme quantiles estimators of Weibull distributions. Journal of Statistical Planning and Inference, 138, 1389-1401.

[15] Falk, M., Hüsler, J. and Reiss, R.D. (2004). Laws of small numbers: Extremes and rare events. 2nd edition, Birkhäuser. 
[16] Gangopadhyay, A.K. (1995). A note on the asymptotic behavior of conditional extremes. Statistics and Probability Letters, 25, 163-170.

[17] Gardes, L. and Girard, S. (2005). Estimating extreme quantiles of Weibull tail-distributions. Communication in Statistics - Theory and Methods, 34, $1065-1080$.

[18] Geluk, J.L. and de Haan, L. (1987). Regular variation, extensions and Tauberian theorems. Math Centre tracts, 40, Centre for Mathematics and Computer Science, Amsterdam.

[19] Girard, S. (2004). A Hill type estimate of the Weibull tail-coefficient. Communication in Statistics - Theory and Methods, 33, 205-234.

[20] Gomes, M.I., de Haan, L., Peng, L. (2003). Semi-parametric estimation of the second order parameter in statistics of extremes. Extremes, 5, 387-414.

[21] Gomes, M.I., Caeiro, F., Figueiredo, F. (2004). Bias reduction of a tail index estimator through an external estimation of the second-order parameter. Statistics, 38, 497-510.

[22] Hall, P. and Tajvidi, N. (2000). Nonparametric analysis of temporal trend when fitting parametric models to extreme-value data. Statistical Science, 15, 153-167.

[23] Hill, B.M. (1975). A simple general approach to inference about the tail of a distribution. Annals of Statistics, 3, 1163-1174.

[24] Kratz, M. and Resnick, S. (1996). The QQ-estimator and heavy tails. Stochastic Models, 12, 699-724.

[25] Loftsgaarden, D. and Quesenberry, C. (1965). A nonparametric estimate of a multivariate density function. Ann. Math. Statist., 36, 1049-1051.

[26] Molinié, G., Yates, E., Ceresetti, D., Anquetin, S., Boudevillain, B., Creutin, J.D. and Bois, P. (2008). Rainfall regimes in a mountainous Mediterranean region: Statistical analysis at short time steps. submitted.

[27] Padoan, S., Ribatet, M. and Sisson, S. (2008). Likelihood-based inference for max-stable processes. submitted.

[28] Schultze, J. and Steinebach, J. (1996). On least squares estimates of an exponential tail coefficient, Statistics and Decisions, 14, 353-372.

[29] Smith, R. L. (1989). Extreme value analysis of environmental time series: an application to trend detection in ground-level ozone (with discussion). Statistical Science, 4, 367-393.

[30] Stone, C. (1977). Consistent nonparametric regression. Annals of Statistics, 5, 595-645.

[31] Weissman, I. (1978). Estimation of parameters and large quantiles based on the $k$ largest observations. Journal of the American Statistical Association, 73, 812-815. 


\section{Acknowledgment}

This work was supported by the Agence Nationale de la Recherche (French Research Agency) through its VMC program (Vulnérabilité: Milieux, Climats). The authors are grateful to Caroline Bernard-Michel and Gilles Molinié for their help in the application to rainfall data. 


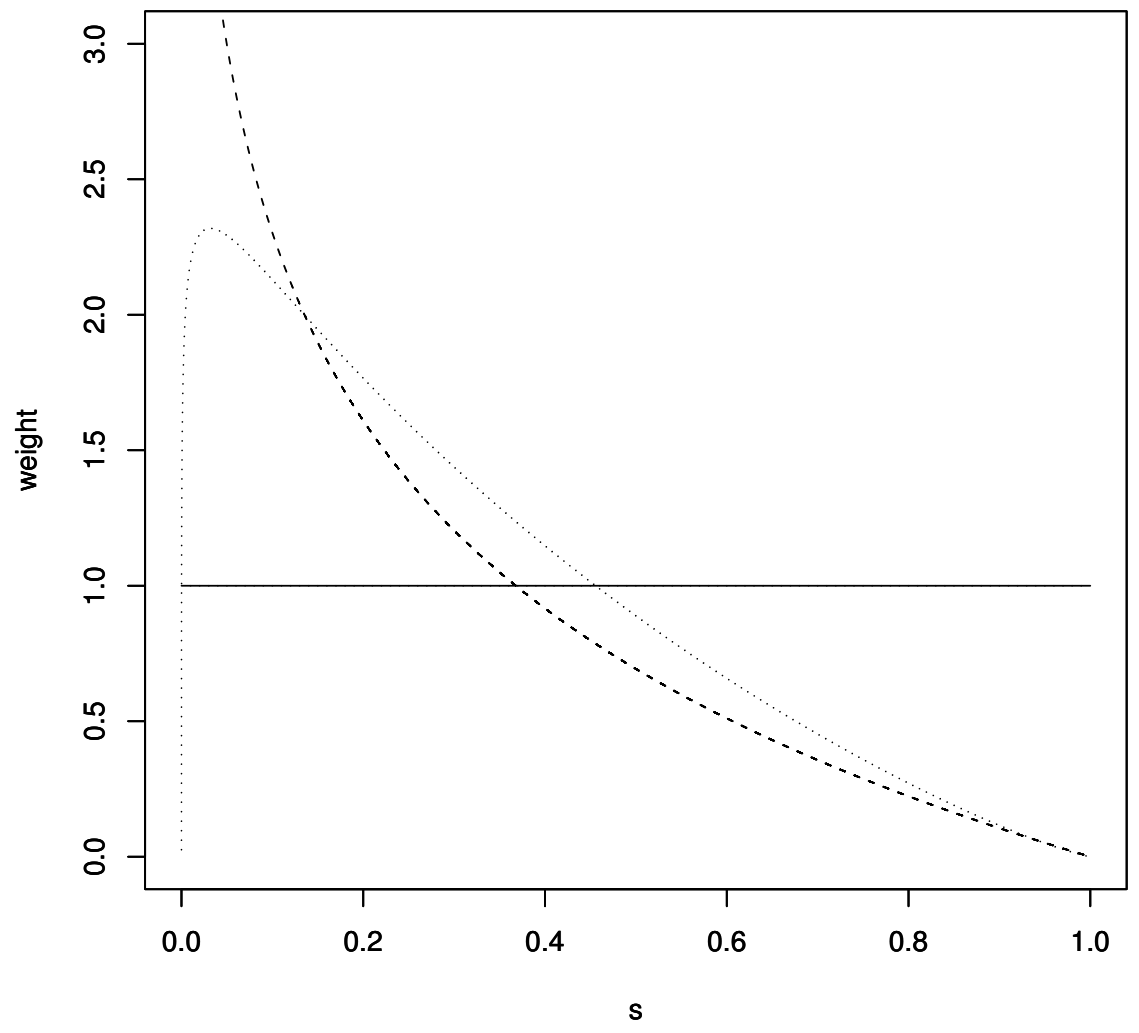

Figure 1: Log-gamma densities associated to $\hat{\gamma}_{n}^{\mathrm{H}}$ (full line), $\hat{\gamma}_{n}^{\mathrm{Z}}$ (dashed line) and $\hat{\gamma}_{n}^{\pi}$ (dotted line). 


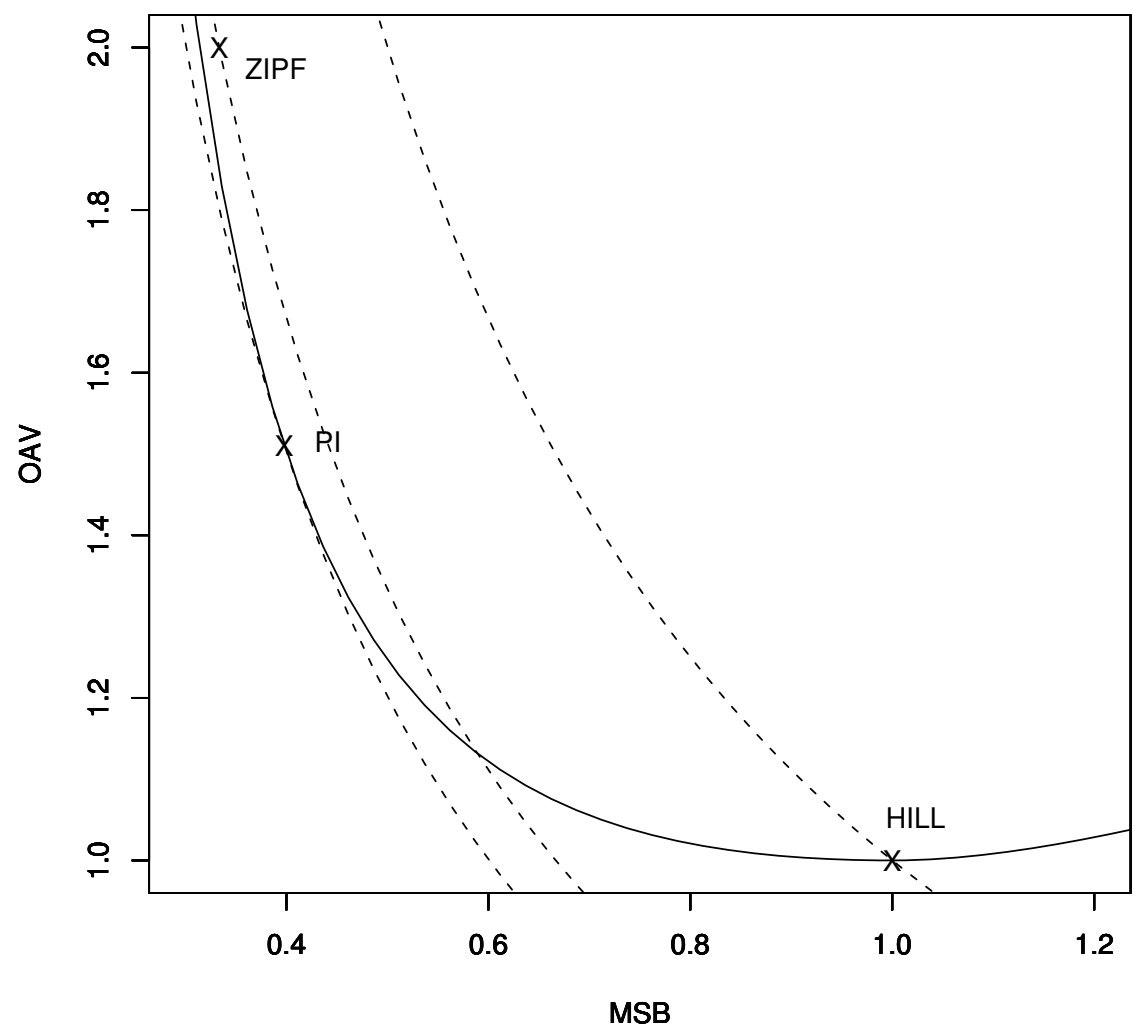

Figure 2: Optimal asymptotic variance $(\mathcal{O} \mathcal{A V})$ as a function of the meansquared bias $(\mathcal{M S B})$ (full line). Dashed lines represent some level curves of $\pi=\mathcal{A} \mathcal{V} \times \mathcal{M S B}$. The points represent the positions of the estimators $\hat{\gamma}_{n}^{\mathrm{H}}$ (HILL), $\hat{\gamma}_{n}^{\mathrm{Z}}(\mathrm{ZIPF})$ and $\hat{\gamma}_{n}^{\pi}(\mathrm{PI})$. 


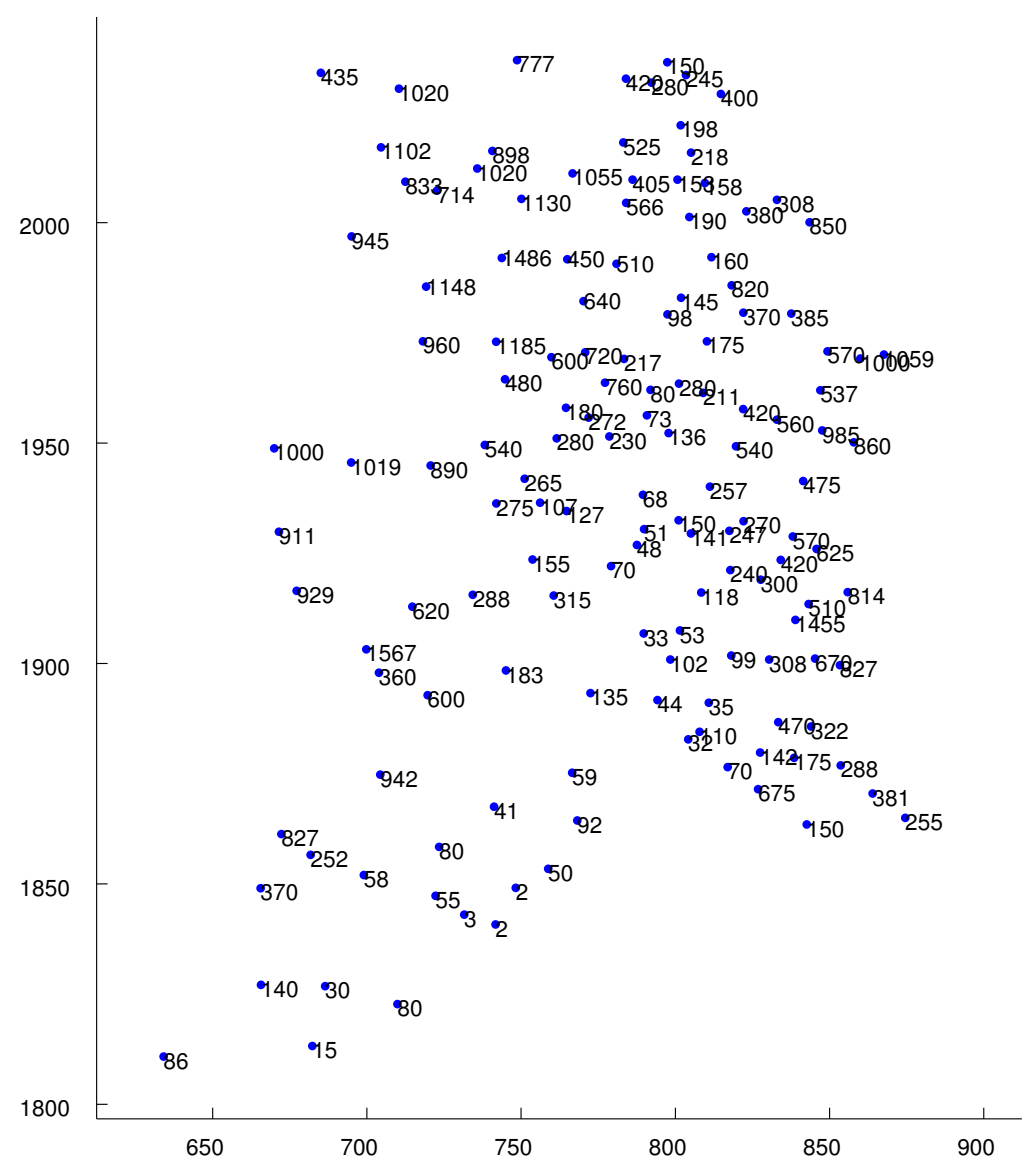

Figure 3: Geographical coordinates (longitude, latitude and altitude) of the 142 raingauges stations. 


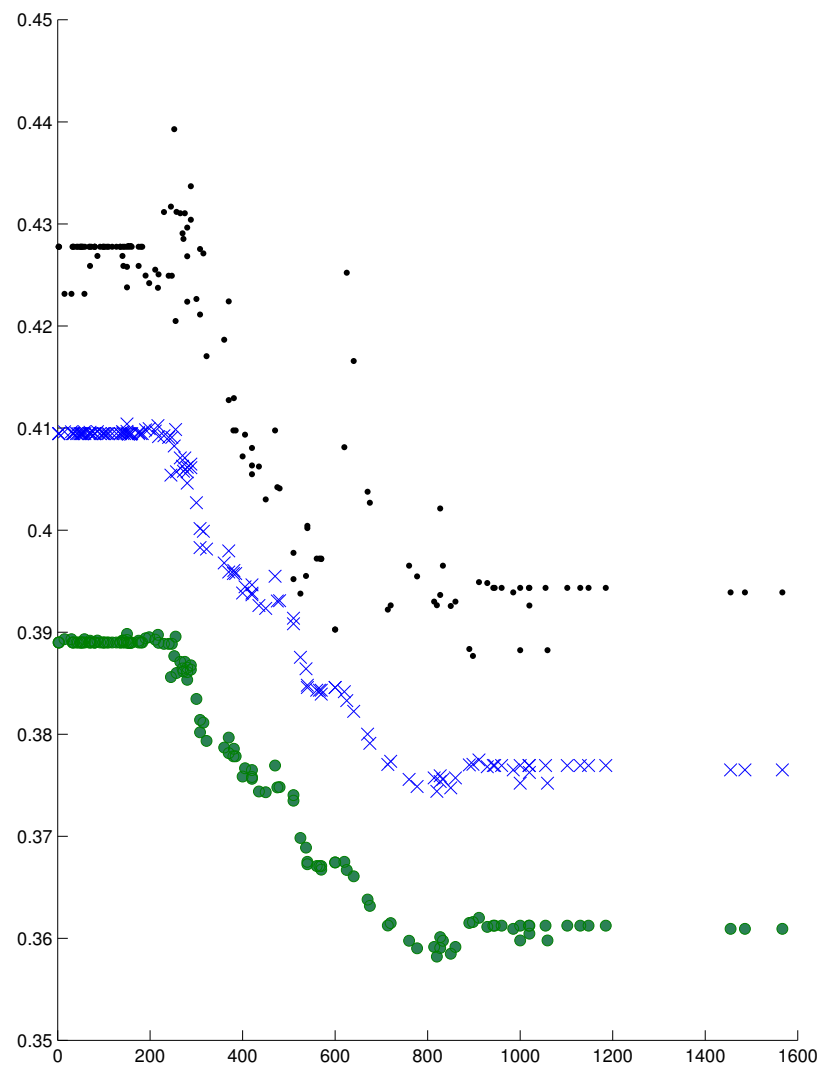

Figure 4: Estimated tail-index as a function of the altitude: $\hat{\gamma}_{n}^{\mathrm{H}}(\cdots), \hat{\gamma}_{n}^{\pi}(\times \times \times)$ and $\hat{\gamma}_{n}^{\mathrm{Z}}(\bullet \bullet \bullet)$. 


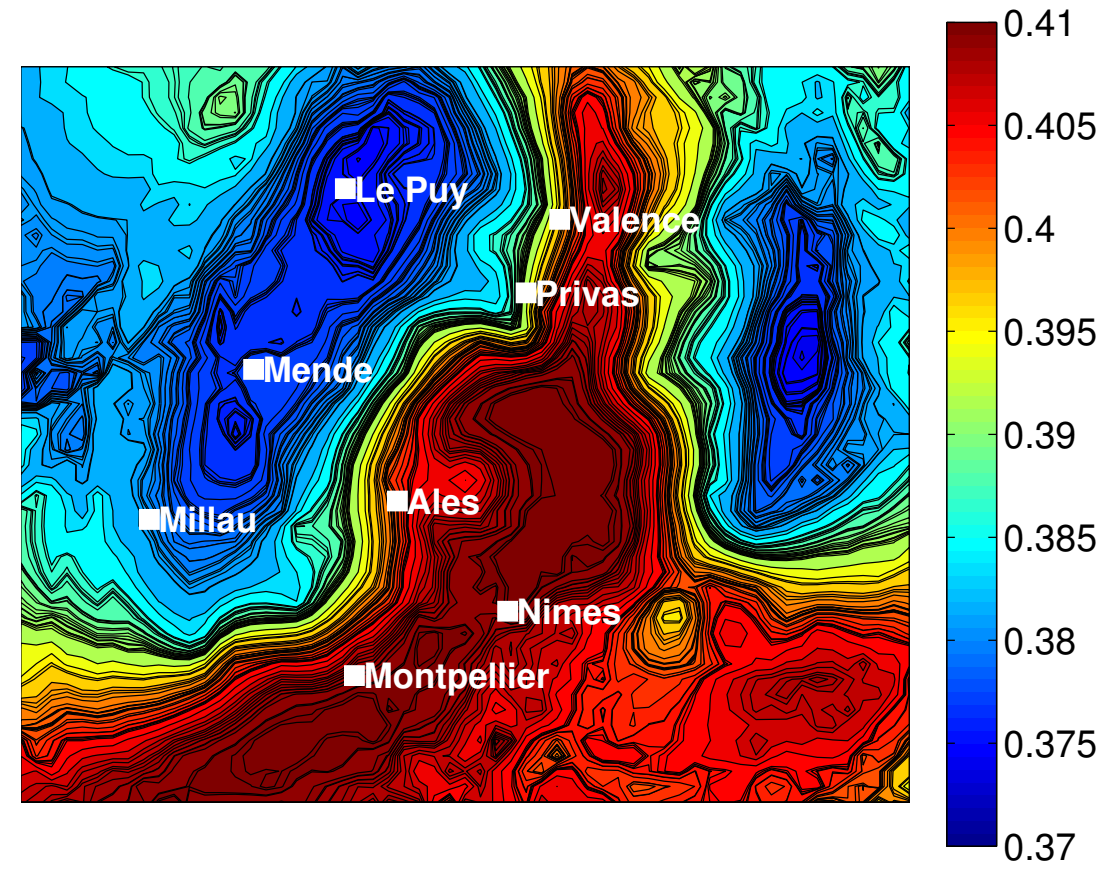

Figure 5: Estimated tail-index $\hat{\gamma}_{n}^{\pi}$ as a function of the longitude and latitude. 


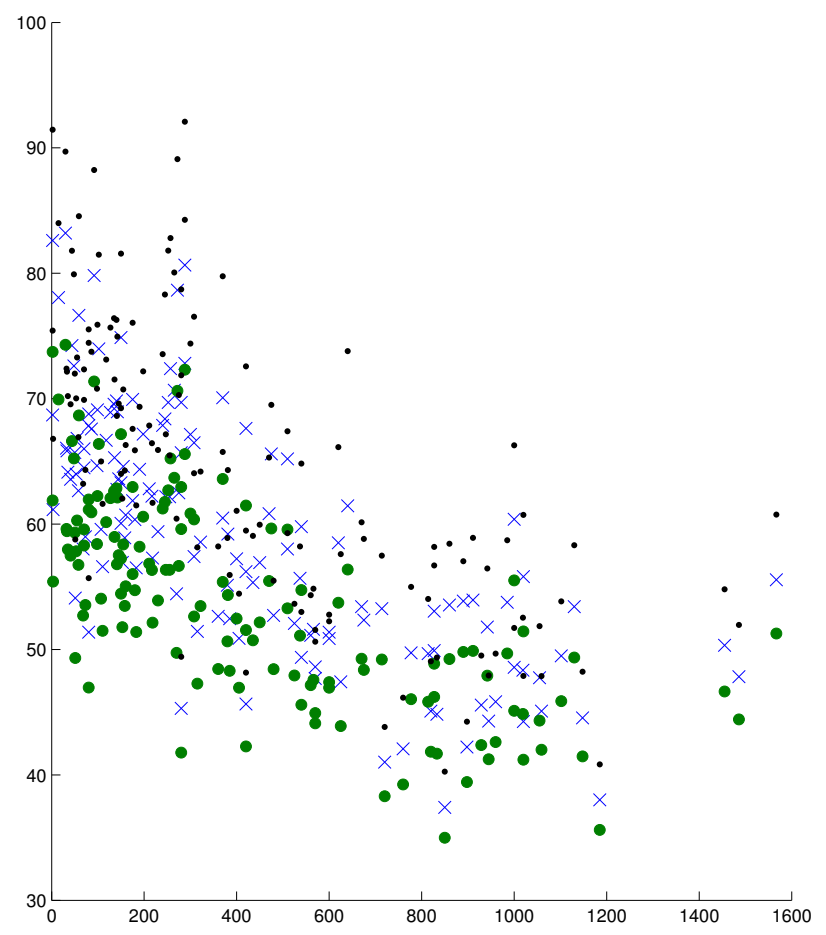

Figure 6: Estimated 10 years- return level (in meters) as a function of the altitude: $\hat{q}^{\mathrm{H}}(\cdots), \hat{q}^{\pi}(\times \times \times)$ and $\hat{q}^{\mathrm{Z}}(\bullet \bullet \bullet)$. 


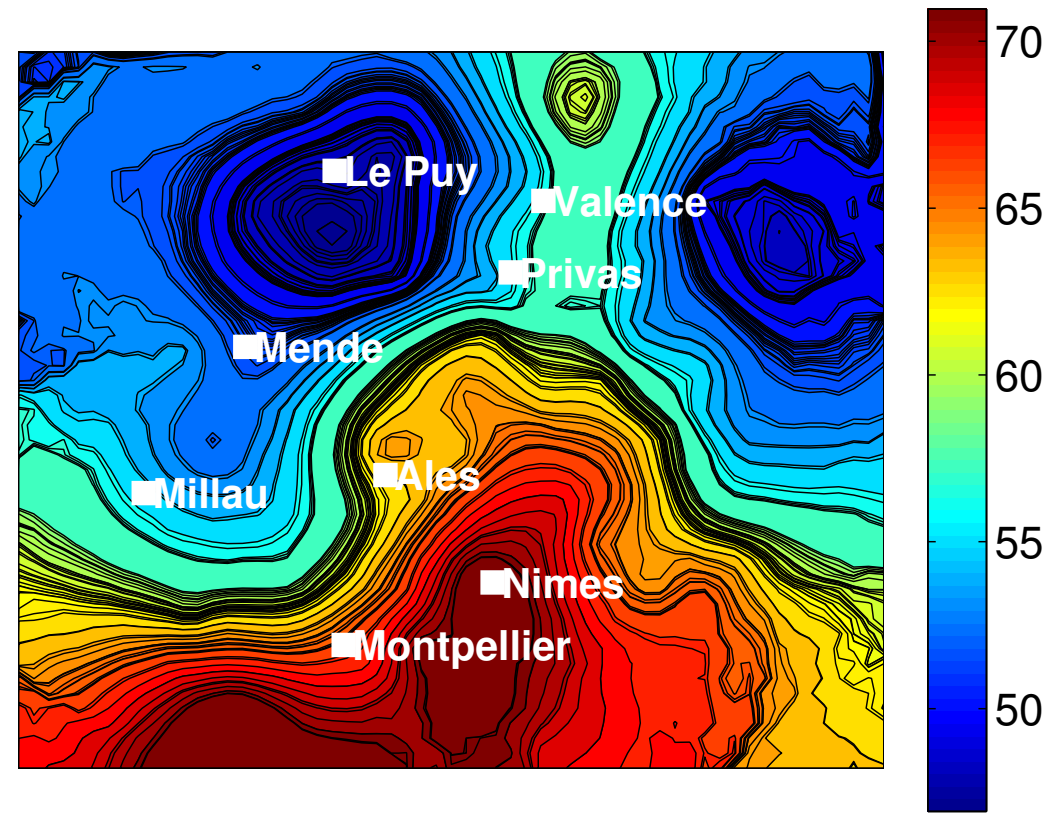

Figure 7: Representation of the 10 years- return level (in meters), estimated with $\hat{q}^{\pi}$, as a function of the longitude and latitude. 